\title{
Power Optimization of Configurable Logic Block in FPGA via Controlling Logic State of Virtual Ground Voltage
}

\author{
Shoaib Shokuhi \\ Islamic Azad University, Science and Research branch (Kurdistan), Department of Electrical Engineering, Tehran, Iran \\ Email: Shoaib.shokuhi@yahoo.com

\begin{abstract}
Ali Hosseini
Islamic Azad University, Science and Research branch (Kurdistan), Department of Electrical Engineering, Tehran, Iran Email: Alihosseini287@gmail.com*
\end{abstract}

\begin{abstract}
In this article, power optimization is investigated in Configurable Logic Block (CLB) of Field Programmable Gate Array (FPGA) for 65nm technology via controlling Virtual Ground Voltage $\left(\mathrm{V}_{\text {ssv }}\right)$ state that follows Power-Gated standard. Initially different Configurable Logic Block are designed through the logic gates and then expanded via adding Look Up Table circuit (LUT) in inputs; afterwards, the samples of Configurable Logic blocks are investigated in two logic states of Virtual Ground Voltage $=0$ and Virtual Ground Voltage $=1$ regarding the power dissipation; whereas $100 \mu \mathrm{s}$ is time reference for simulation of time controller of Virtual Ground Voltage function. First Configurable Logic Block are kept at logic state of Virtual Ground Voltage $=l$ (power gated) for $10 \mu \mathrm{s}$ out of $100 \mu \mathrm{s}$ and remaining time at logic state of Virtual Ground Voltage $=0$ (power not gated); then the simulation test is repeated up to $50 \mu \mathrm{s}$ in 5 steps for each Configurable Logic Block sample. Finally the result shows that reduction being at logic state of Virtual Ground Voltage $=0$ in a constant time period has linear effect on decreasing average power. With the Configurable Logic Block in operation for 50\% of the total time in Virtual Ground Voltage $=1$ logic state, the average power reduces up to $49 \%$ in the best case scenario. Meanwhile the Configurable Logic Block can still preserve its logic state.
\end{abstract}

Index Terms - Power optimization, logic gates, Field Programmable Gate Array, simulation, power gated, look up Table, Configurable Logic Block, Virtual Ground voltage.

\section{INTRODUCTION}

Increased usage of FPGA in portable devices and becoming smaller in size with every generation process, causes increase in power consumption [1] thus, structure should be modified through presenting new methods and modification of internal block for reducing power. In a digital complementary metal-oxide-semiconductor (CMOS) circuit, dynamic power dominates the total- power dissipation. Reducing the supply voltage $V_{d d}$ is the most effective approach to reduce dynamic power dissipation. Lowering $V_{d d}$ is also important in deep submicron (DSM) technologies to avoid reliability problems [2]. However, reducing the supply voltage alone causes serious degradation in the circuit's performance. One way to maintain performance is to scale down both Vdd and the threshold voltage Vth. However, reducing Vth increases the subthreshold leakage current exponentially [3]. Integrated low-power architecture is proposed and implemented for FPGA, which fully utilizes the fine-grain assignment of VDD/VTH in time and space that way, 4 Configurable Logic Blocks (CLB) are grouped into one block where is shared [4]. In [5] average switching activity of the input and output state variables is reduced by minimizing the number of bit changes during state transitions. In [6] a variable threshold voltage as keeper circuit techniques proposed for simultaneous power reduction. Power gating [7]-[8] is the most popular circuit technique to suppress subthreshold leakage. It consists of gating, or cutting off, a circuit from its power supply rails during standby mode. When footer, located between a logic block and $V_{s s}$, is turned off, the voltage at virtual ground $\left(V_{s v v}\right)$, where footer has its drain, rises slowly until it reaches a steadystate potential, which is usually close to $V_{d d}$. Similarly, if a header is used and if it is turned off, the voltage at virtual $V_{d d}\left(V_{d d v}\right)$, slowly goes down to a steady-state potential, which is close to $V_{s s}$. Due to collapse of either $V_{s s v}$ or $V_{d d v}$ during standby, the circuit states that are represented by sequential elements and primary outputs have to be captured in advance and preserved. Data retention elements (flip-flops and isolation circuits) are used to preserve circuit states during standby mode, if the states are needed again after wake-up. These elements must be controlled by an external power management unit, causing a network of control signals implemented with extra wires and buffers. A power-gated circuit with autonomous data retention (APG) is proposed in [9] to remove the overhead involved in control signals. Retention elements in APG derive their control by detecting rising potential of virtual ground rails when 
power gating starts, i.e., they control themselves without explicit control signals. APG and standard PG are shown in figure 1. In both circuits, combinational gates are placed between $V_{d d}$ and $V_{s s v}$. $V_{s s v}$ gets controlled through footer, that way it is powered on, in an active state and powered off in a standby state. In APG [9], storing Flip flop and isolation circuits are replaced with Autonomous Retention Flip Flop (ARF) and Autonomous Retention Isolation (ARI). ARF and ARI are used for storing data while footer is off. For this purpose one slave latch, which is in charge of ongoing data, is connected directly to $V_{s s}$ and $\mathrm{V}_{\mathrm{dd}}$ to capture Flip flop data [9]-[10]. In [11] area-efficient circuits for programmable fine-grained power-gating of individual unused interconnect switches is presented. Fine-grain power gating is more effective than coarse-grain power gating to reduce the active leakage power of unused logic and interconnection resources [12]. In [13] a modification to the fabric of an FPGA is presented that enables dynamically-controlled power gating, in which logic clusters can be selectively powered-down at run-time. Detecting the data arrival in advance prevents the delay increase for waking-up and the power consumption of unnecessary power switching [14]. In [15] architecture is presented that enables selectively powering down SBs along with the logic blocks during their idle periods. In [16] a tool is presented that capable of modeling the power usage of many different field programmable gate array (FPGA) architectures. In [17] a novel directional coarse grained power gating architecture for switch boxes is presented. In [18] an FPGA architecture is presented that enables dynamically controlled power gating, in which FPGA resources can be selectively powered down at run-time. In [19] area, delay, and energy for two intra-CLB topologies are compared. Figure 2 shows how logic blocks get controlled through $V_{s s v}$ state via footer switch. In this paper by controlling time period of $V_{s s v}$ logic state through Footer switch, power dissipation in CLB block is investigated. First in section 2, power consumption for 5 sample circuits of CLB block is investigated. In section 3, we apply our method for reduction of power dissipation and test it through the sample CLB blocks; finally in section 4 we show result of power recovery in the sample CLB blocks. In this paper when we mention $V_{s s v}=1$ or 0 , we describe $V_{s s v}$ logic state, not its voltage.

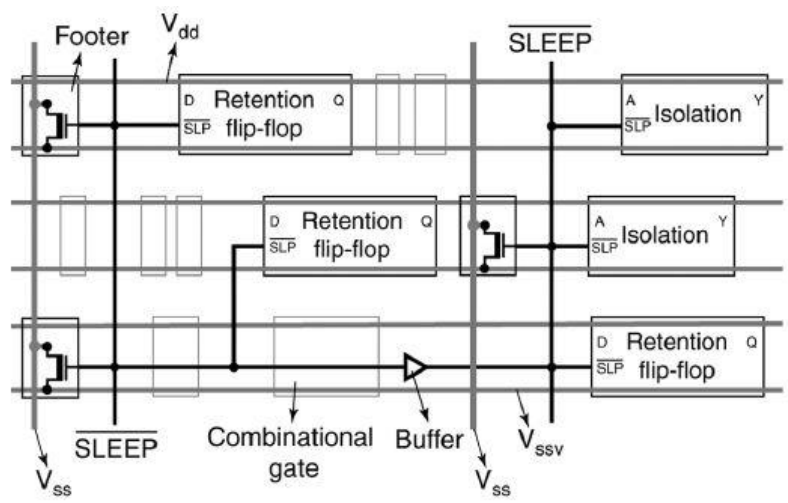

(a)

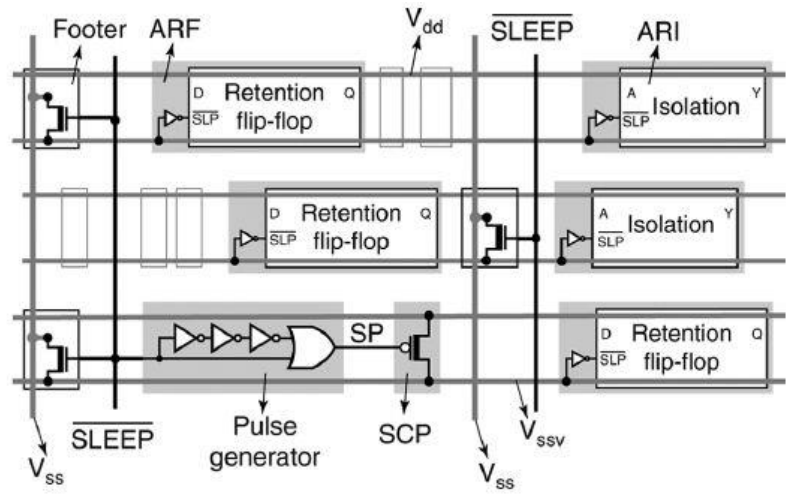

(b)

Fig.1. a. Standard PG [9] and b. APG [9]

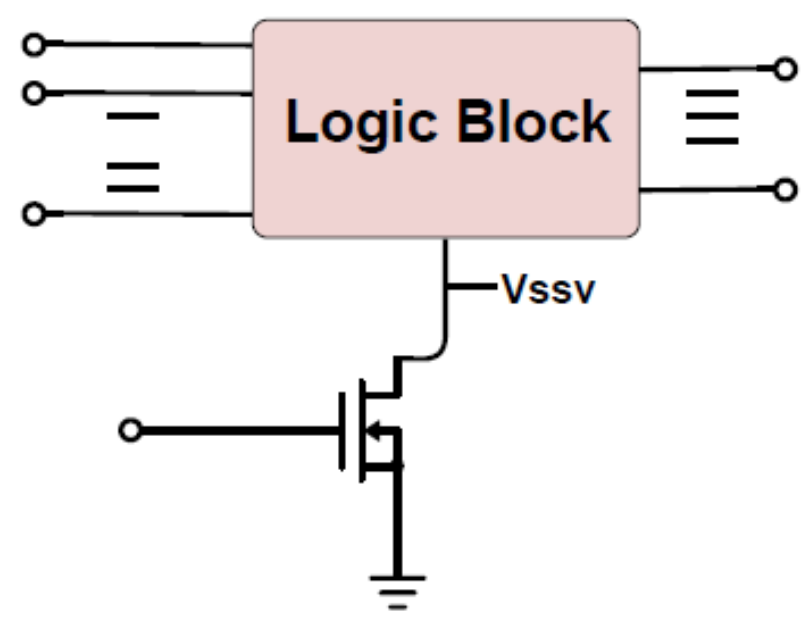

Fig.2. Footer switch in logic block

\section{METhod of POWER CONSUMPTION Simulation ON 5 SAMPLES OF CLB BLOCKS}

\section{A. Clb Design through Transistor Model of Logic Gates}

Basic logic gates (NOT, AND, OR,...) are simulated through NMOS and PMOS model in 65nm technology for design of CLB block in FPGA. Structure of CMOS model for Inverter, 2-input NAND, 2-input NOR and SR Flip flop, is used in this research, shown in figure 3.

Utilizing these CMOS transistor models, we are able to design the main blocks in FPGA, since architecture of CLB block is made through Logic gates. Initially in the simulation process, Flip flop is designed through combination of transistor model of (OR), (AND) and (NOT) gates. Then through synthesis of two (AND), one (NOT) and one (OR), (MUX-2 input) is acquired (depending on amount of inputs in MUX). Afterward via utilizing the logic gates, (LUT) is modeled and in result by using MUX, LUT and F.F, CLB block, which is most important block in FPGA, is obtained. Following which, power dissipation of each logic gate, which contributes in CLB block design, is investigated. 


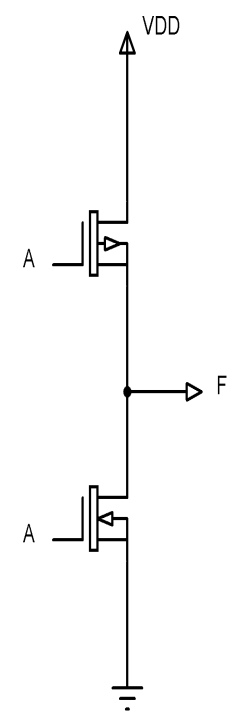

INVERTER

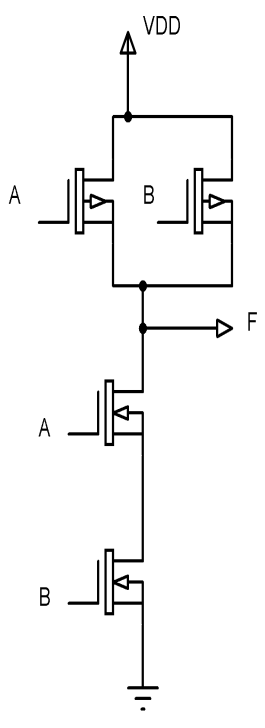

2-INPUT NAND
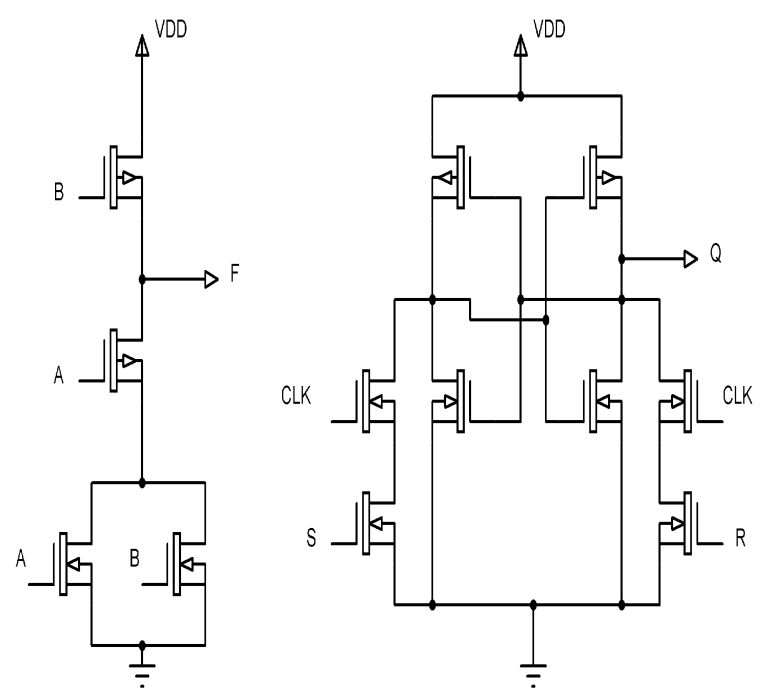

2-INPUT NOR

\section{FLIPFLOP}

Fig.3. CMOS transistor model used in logic gates and flip-flop for design of the CLB block

\section{B. Power Equations}

Allowable power consumption is main concern while expanding integration in circuits as well as processing speed. Depending on the design, different formulas can be considered for power dissipation. When it is required to assign voltage and current in power supply, peak power factor is more useful, when battery consumption and cooling system is the main concern, then $P_{a v}$ factor becomes a preferable parameter that is shown in (1) [20].

$$
w=\frac{1}{T} \times \int_{0}^{t} p(t) d t=\frac{V_{\text {sup }}}{T} \times \int_{0}^{t} I_{\text {sup }}(t) d t
$$

In formula (1), $P(t)$ is transition power, $I_{\text {sup }}$ is the amount of current that is drawn from $V_{\text {sup }}$ in period of $\sum[0, t]$.

Table 1. Power dissipation for logic gates, Flip flop, Mux and CLB

\begin{tabular}{|c|c|}
\hline Gate & Power Dissipation \\
\hline NOT & $18 \mu \mathrm{w}$ \\
\hline NAND & $60 \mu \mathrm{w}$ \\
\hline XOR & $1 / 2 \mathrm{mw}$ \\
\hline FLIP FLOP & $600 \mu \mathrm{w}$ \\
\hline MUX & $600 \mu \mathrm{w}$ \\
\hline LUT & $600 \mu \mathrm{w}$ \\
\hline CLB TEST & $70 \mathrm{mw}$ \\
\hline
\end{tabular}

After acquiring power dissipation, in proportion with given input and transistor CMOS model for each gate, final results are concluded in table 1. Table 1 shows average power $\left(P_{a v}\right)$ of logic gates, Flip flop, Mux, LUT, CLB.

\section{Implementation of Sample Circuits on CLB Block}

In figure 4 , internal schematic of CLB block, which designed through logic gates and flip flop, is shown that includes LUT at input, MUX in middle and Flip flop at output. The main CLB block is utilized as the first sample circuit under test. In this paper 5 sample circuits are presented on basis of the CLB block shown in figure4; in each one, 2 logic gates are added to LUT section of last sample circuit in order to investigate and compare power dissipation on these sample circuits.

Table 2. Added logic gates to LUT of the sample CLB circuits

\begin{tabular}{|c|c|c|c|c|c|c|}
\hline & \multicolumn{5}{|c|}{ The 5 samples of CLB blocks } \\
\hline & & $\mathbf{I}$ & II & III & IV & $\mathbf{V}$ \\
\hline \multirow{12}{*}{ 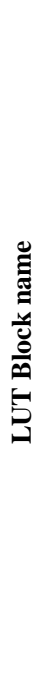 } & G1 & XOR & XOR & XOR & XOR & XOR \\
\hline & F1 & OR & OR & OR & OR & OR \\
\hline & G2 & NAND & NAND & NAND & NAND & NAND \\
\hline & F2 & AND & AND & AND & AND & AND \\
\hline & G3 & & AND & AND & AND & AND \\
\hline & $\mathbf{F 3}$ & & XOR & XOR & XOR & XOR \\
\hline & G4 & & AND & AND & AND & AND \\
\hline & F4 & & & OR & OR & OR \\
\hline & G5 & & & & NAND & NAND \\
\hline & F5 & & & & OR & OR \\
\hline & G6 & & & & & XOR \\
\hline & F6 & & & & & NAND \\
\hline
\end{tabular}

According to internal architecture of designed CLB block in figure (4), logic gates are added to initial LUT section as presented in table 2. Accordingly in each sample circuit, one LUT block includes 2 logic circuits are added to last sample circuit so that from 4 input gates 
in first sample circuit, it is expanded to 12 input gates in 5th circuit. In figure (4) respectively XOR-OR-NANDAND are used in LUT section .

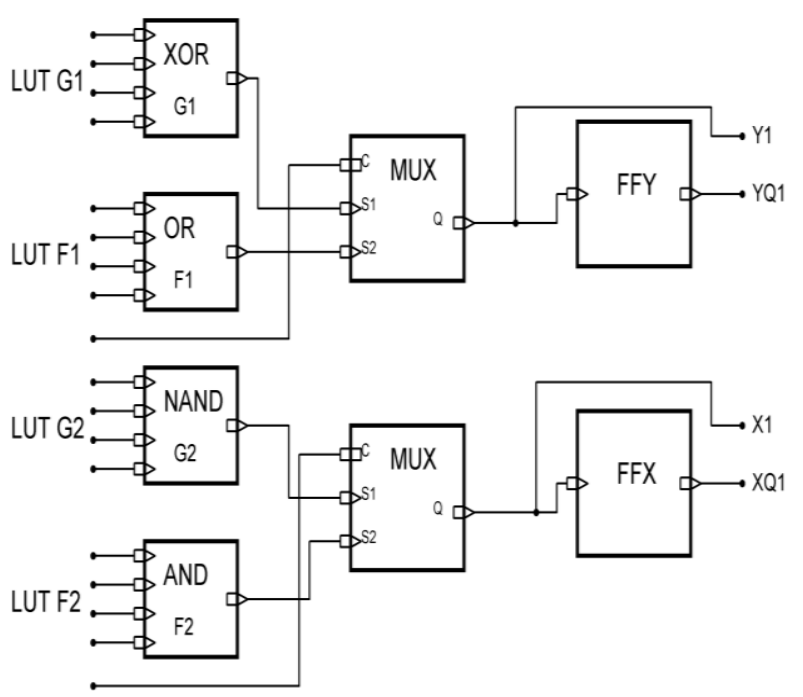

Fig.4. Design of CLB block through the logic gates (I sample CLB)

\section{Dissipation Power in First Sample Circuits}

Main CLB block architecture is utilized as the first sample circuit under test. In this test, dissipation power is investigated through simulation in two logic state of $V_{s s v}=0$ and $V_{s s v}=1$. Results in Table 3 shows that dissipation power is reduced in $V_{s S v}=1$ state.

\section{E. Dissipation Power in 5th Sample Circuits}

In $5^{\text {th }}$ sample circuit, else LUT is added to $4^{\text {th }}$ sample circuit includes (G6(XOR)-F6(NAND)) and totally compared to main CLB circuit, 4 LUT include ((G3(AND), F3(XOR), G4(AND), F4(OR), G5(NAND), F5(OR), G6(XOR), F6(NAND)) is added to inputs that shown in figure 5 . The power dissipation of $5^{\text {th }}$ sample circuit, in two logic states of $V_{s s v}=0$ and $V_{s s v}=1$, is shown in table $3(\mathrm{~V})$. According to obtained result, despite expanding CLB block through added LUT, dissipation power reduced dramatically via keeping $V_{s s v}$ at state of logic 1 .

Table 3. Dissipation power of sample circuits of CLB at logic state of $\mathrm{V}_{\mathrm{ssv}}=1\left(P_{\text {Gated }}\right)$ and $\mathrm{V}_{\mathrm{ssv}}=0\left(P_{\text {not Gated }}\right)$

\begin{tabular}{|c|c|c|c|c|c|}
\hline $\begin{array}{c}\text { Logic } \\
\text { State }\end{array}$ & I & II & III & IV & V \\
\hline $\begin{array}{c}\mathbf{V}_{\text {ssv }}=\mathbf{0} \\
\left(\boldsymbol{P}_{\text {Gated }}\right)\end{array}$ & $75 \mathrm{mw}$ & $125 \mathrm{mw}$ & $165 \mathrm{mw}$ & $205 \mathrm{mw}$ & $250 \mathrm{mw}$ \\
\hline $\begin{array}{c}\mathbf{V}_{\text {ssv }}=\mathbf{1} \\
\left(\boldsymbol{P}_{\text {notGated }}\right)\end{array}$ & $350 \mathrm{uw}$ & $525 \mathrm{uw}$ & $700 \mathrm{uw}$ & $870 \mathrm{uw}$ & $1.8 \mathrm{mw}$ \\
\hline
\end{tabular}

\section{F. Dissipation Power Results in Sample Circuits}

In table3, power dissipation for 5 sample circuits in two logic states of $V_{s s v}=1$ and $V_{s s v}=0$ is compared and shows despite expanding LUTs in CLB, at state of $V_{s s v}=1$ (Power gated) power is reduced.
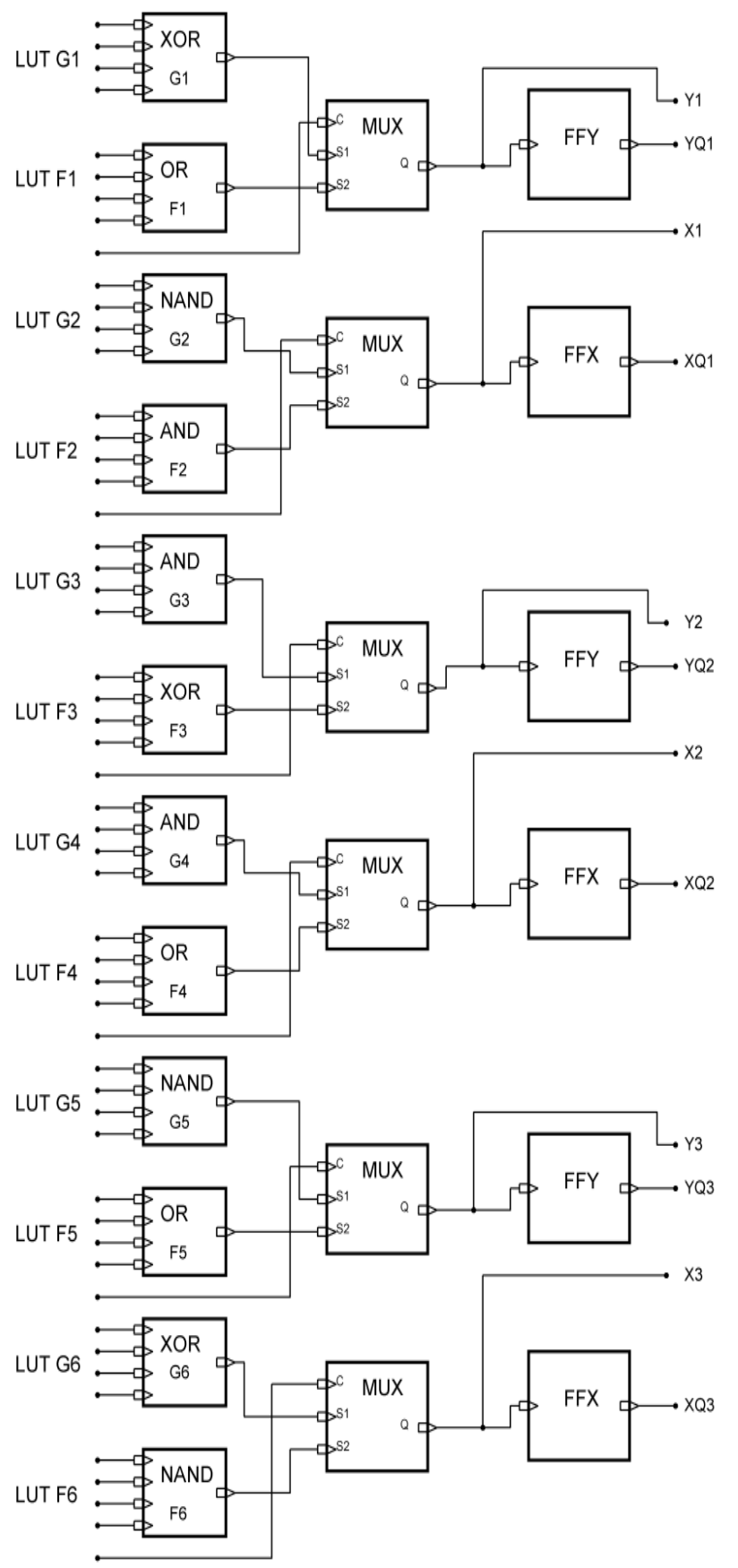

Fig.5. V $\left(5^{\text {th }}\right)$ sample circuit of CLB block

\section{OUR PROPOSED METHOD FOR POWER RECOVERY}

According to obtained results, we analyze effect of $P_{\text {Gated }}$ (power consumption in power-Gated state)and $P_{n o t}$ Gated (power consumption in full function state) in the CLB blocks by time controller of $V_{s s v}$ logic state and evaluate it through power recovery factor in different CLB blocks. First $100 \mu$ s is considered as a time reference for state of $V_{s s v}$ in 5 samples of CLB blocks then according to time intervals out of $100 \mu \mathrm{s}$ power get evaluated in power gated $\operatorname{state}\left(V_{s s v}=1\right)$. Below formula is used for calculation of power dissipation in CLB samples. In equation $2, T$ is Time reference and is equal to 
$T=100 \mu s$ and (per) is the period for which $V_{s s v}=1$. Time period for the logic states of $V_{s s v}=1$ and $V_{s s v}=0$ is shown in figure 6.

$$
P_{\text {Gated }}=\left\{\left(\frac{\text { per }}{T} \times P_{\text {Gated }}\right)+\left(\left(\left|\frac{\text { per }-T}{T}\right|\right) \times P_{\text {notGated }}\right)\right\}
$$

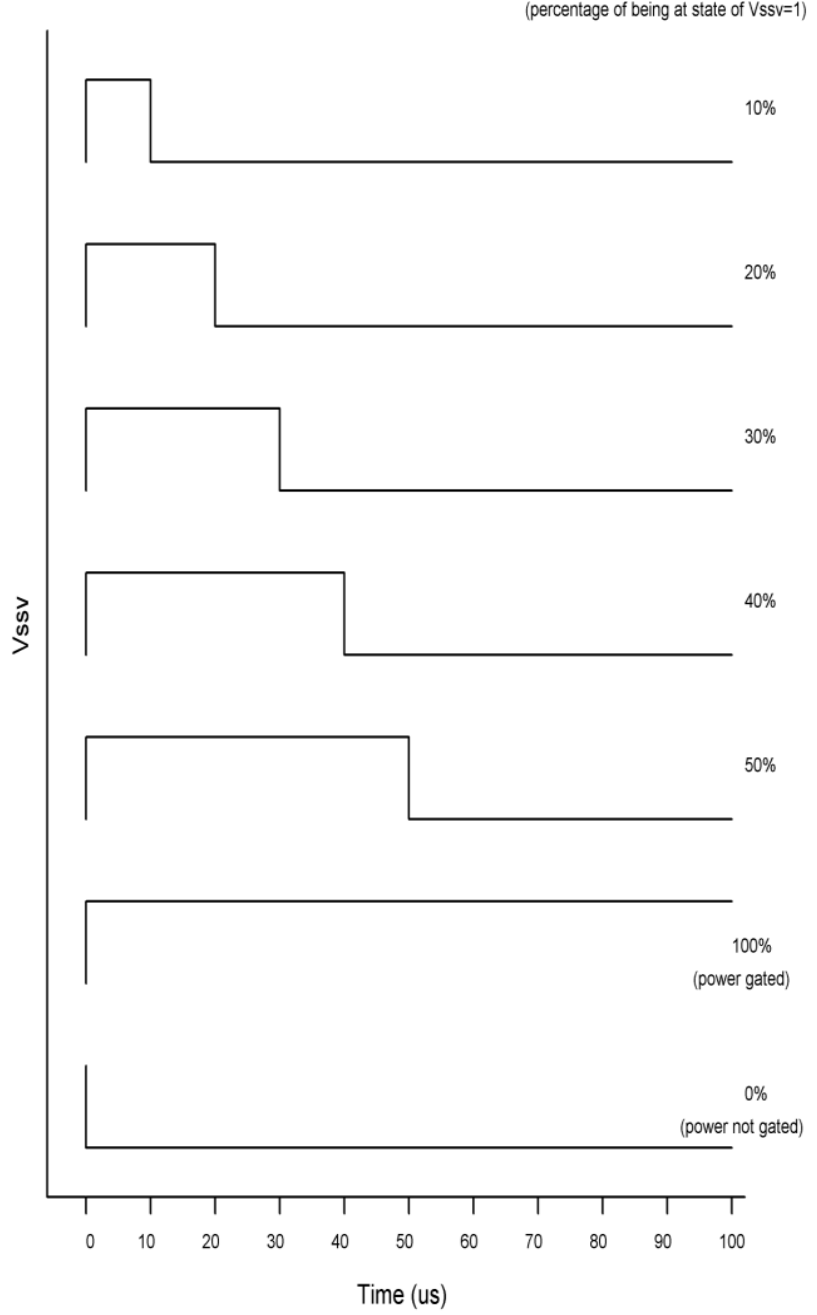

Fig.6. Time period for the logic states of $\mathrm{Vssv}=1$ and $\mathrm{Vssv}=0$ in the method

\section{RESULT}

In table 4 power dissipation is considered in column 2 up to 6 in (per) interval for $10 \mu \mathrm{s}$ up to $50 \mu \mathrm{s}$ out of $100 \mu \mathrm{s}$ in 5 steps for sample circuits of CLB. Column 7 and 8 respectively show power dissipation in power gated state and power not gated state.

According to figure 7, as much as (per) period (period of time that $V_{s s v}=1$ ) gets longer, power dissipation get less, thus The power reduction is in linear proportion with (per); in addition, slope of lines become more along with expanding the sample circuits of CLB.
Table 4. Power dissipation for different percentages of being at state of $\mathrm{P}_{\text {gated }}\left(V_{s s v}=1\right)$ out of $100 \mu s$ and $\mathrm{P}_{\text {not gated }}\left(0 \%\right.$ out of $100 \mu s$ at state of $V_{s s v}$ $=1$ ) in the CLB sample circuits.

\begin{tabular}{|c|c|c|c|c|c|c|c|}
\hline No. & $\begin{array}{c}\mathbf{1 0 \%} \\
\mathbf{M w}\end{array}$ & $\begin{array}{c}\mathbf{2 0 \%} \\
(\mathbf{M w})\end{array}$ & $\begin{array}{c}\mathbf{3 0 \%} \\
(\mathbf{M w})\end{array}$ & $\begin{array}{c}\mathbf{4 0 \%} \\
(\mathbf{M w})\end{array}$ & $\begin{array}{c}\mathbf{5 0 \%} \\
(\mathbf{M w})\end{array}$ & $\begin{array}{c}\mathbf{P}_{\text {gated }} \\
\mathbf{1 0 0 \%} \\
(\boldsymbol{\mu W})\end{array}$ & $\begin{array}{c}\mathbf{P}_{\text {notgated }} \\
\mathbf{0 \%} \\
(\mathbf{M w})\end{array}$ \\
\hline I & 67 & 60 & 52 & 45 & 37 & 350 & 75 \\
\hline II & 112 & 100 & 87 & 75 & 62 & 525 & 125 \\
\hline III & 148 & 132 & 115 & 99 & 82 & 700 & 165 \\
\hline IV & 184 & 164 & 143 & 123 & 102 & 870 & 205 \\
\hline V & 225 & 200 & 175 & 150 & 125 & 1800 & 250 \\
\hline
\end{tabular}

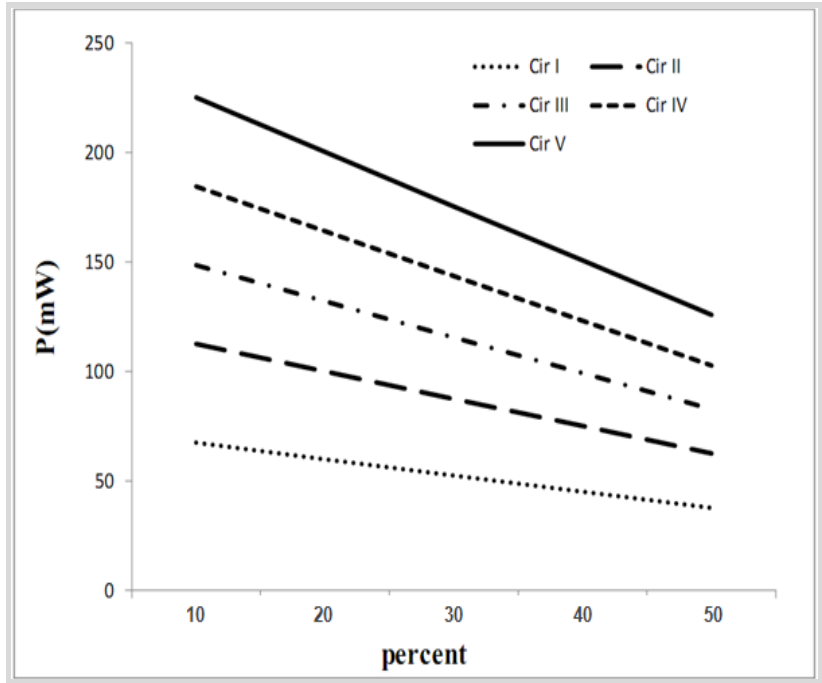

Fig.7. Reducing power according to percentage of period of time that Vssv=1 out of $100 \mu$ s in 5 sample circuits of CLB

Power Recovery is a factor that indicates power reduction in sample circuits of CLB block. Result in table 5 shows being in logic state of $V_{s s v}=1$, raises Recovery power factor. Moreover row I to $\mathrm{V}$ shows power recovery between $10 \%$ to $50 \%$ in (per) (period of time that $V_{s s v}=1$ out of $100 \mu \mathrm{s})$. $(A v g)$ shows average power recovery in 5 sample circuits. Linear relation of Average power reduction versus per ((percent of time out of $100 \mu \mathrm{s}$ that $V s s v=1)$ is presented in Figure 8. It indicates that despite number of LUT used in CLB block, lowering activity of $V_{s s v}$ (being at logic state of $V s s v=1$ for longer) results in, higher power recovery.

$$
\text { power }_{\text {Recovery }}=\left(\frac{P_{\text {notGated }}-P_{\text {Gated }}}{P_{\text {notGated }}}\right) \times 100
$$

For obtaining maximum power saving, it requires to keep $V_{s s v}=1$ for $50 \%$ of total duty cycle that cause $49 \%$ saving in energy but in contrast extra delay will be imposed that limits our method to just low speed application. By using less percentage of duty cycle being at state of $V_{s s v}=1$, energy optimization get reduced but it rises time efficiency and in result it will lead to fast performance, though the method can be adjusted according to the design and application. By using Power 
Recovery factor, it is possible to measure energy saving through controlling duty cycle time of Vssv in operation that can be applied to previous works that requires more power saving and less speed.

Table 5. Power Recovery in the sample circuits of CLB according to percentage of time out of $100 \mu s$ in logic state of $V s s v=1$

\begin{tabular}{|c|c|c|c|c|c|}
\hline \multirow{2}{*}{$\begin{array}{c}\text { Sample } \\
\text { CLB } \\
\text { Circuit } \\
\text { No. }\end{array}$} & \multicolumn{5}{|c|}{$\begin{array}{l}\text { Power recovery }(\mathrm{mw}) \text { according to time } \\
\text { percentage of being at } V s s v=1 \text { state }\end{array}$} \\
\hline & $\begin{array}{l}10 \% \\
(\mathrm{mw})\end{array}$ & $\begin{array}{l}20 \% \\
(\mathrm{mw})\end{array}$ & $\begin{array}{l}30 \% \\
(\mathbf{m w}) \\
\end{array}$ & $\begin{array}{l}40 \% \\
(\mathrm{mw})\end{array}$ & $\begin{array}{l}\mathbf{5 0 \%} \\
(\mathbf{m w})\end{array}$ \\
\hline I & 9.953 & 19.906 & 29.86 & 39.813 & 49.766 \\
\hline II & 9.958 & 19.916 & 29.874 & 39.832 & 49.79 \\
\hline III & 9.957 & 19.915 & 29.872 & 39.830 & 49.787 \\
\hline IV & 9.957 & 19.915 & 29.872 & 39.830 & 49.787 \\
\hline V & 9.928 & 19.856 & 29.784 & 39.712 & 49.64 \\
\hline Avg & 9.950 & 19.901 & 29.852 & 39.803 & 49.754 \\
\hline
\end{tabular}

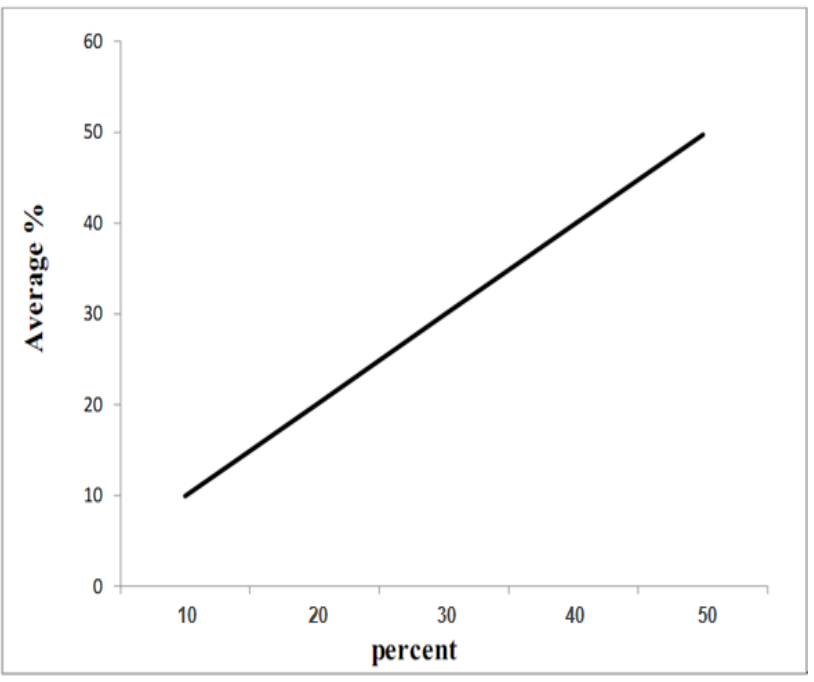

Fig.8. Average power reduction against percentage of being at logic state of $V s s v=1$ out of $100 \mu \mathrm{s}$

\section{RELATED WORK AND DISCUSSION}

Approximately 16 percent of the FPGA power is consumed by the CLBs alone [21]. As technology nodes scale down, the leakage power is going to increase in these logic blocks. Also, its effects on the total power should not be ignored for FPGA cores that can be embedded within an ASIC architecture [21]. The components of the CLB are latches (in place of SRAM cells), Muxes, and flip-flop. The methods considered in previous works are multi-threshold CMOS, clock gating, and variable gate length transistors [21]. Previous work on creating a low energy FPGA, explored many architectures and circuit techniques. [22] The lookup table size was chosen because previous studies revealed that it is a good choice for speed and density. A variable gate length method was introduced to reduce the power consumption of the CLB. Variable gate length transistors allow for the reduction of leakage in circuits that do not require high performance. The clock gating of the D flipflop isn't required to be fast if the flip flop is not switched very frequently. Therefore, the clock gating circuitry can slowly compare the input and output of the flipflop and have a value ready when the flipflop is clocked. This method reduced the power consumption for the sequential mode by reducing the leakage in those gates. The comparison of the three CLB power reduction methods can be seen in Figure 9. The delay of the logic block increases when the clock gating technique is integrated. The addition of the variable gate length transistors only increases the delay slightly but results in a large reduction in power consumption [21]. In this paper power optimization of CLB by utilizing time controlling of virtual ground (Vssv) is investigated and in result, it showed that by CLB in operation for $50 \%$ of the total time in $V s s v=1$ logic state, the average power reduces up to $49 \%$, but depends on application of design, whether, it will allow designer to keep the CLB off for the special period of time. By merging this method with previous works, there will be more potential for reducing power dissipation that can be accomplished in future works.

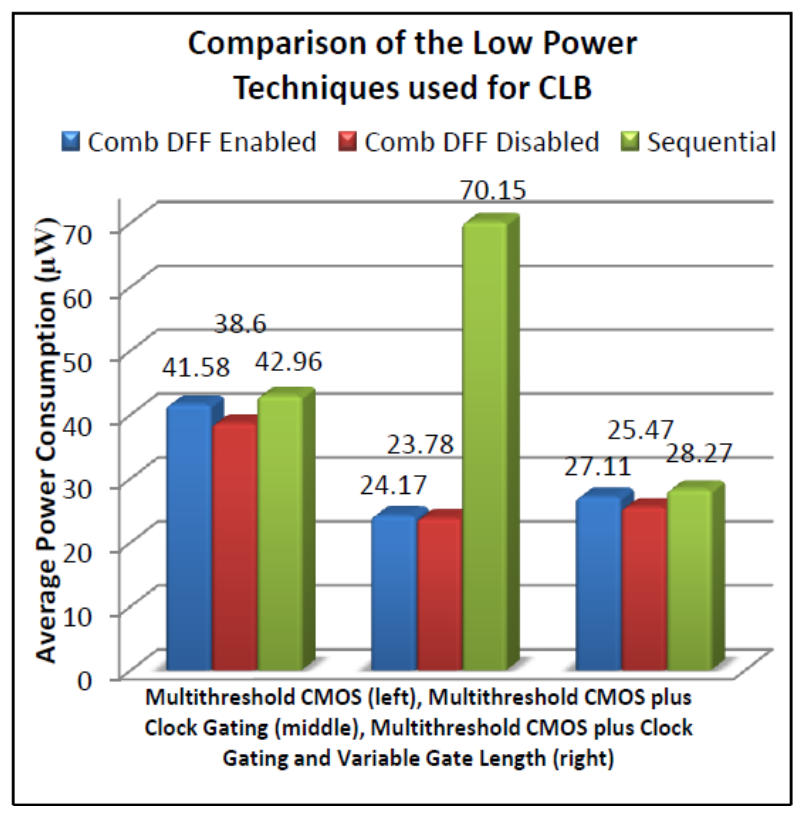

Fig.9. Power of optimized CLB in previous works ( $\mathrm{f}=200 \mathrm{MHZ}$ ) [21]

\section{CONCLUSION}

In this research initially power consumption of logic gates that contribute in CLB block design is obtained, then through investigating internal block of CLB in FPGA at 2 logic states of $V_{s s v}=1$ and $V_{s s v}=0$, reducing power is considered. In result, it is observed that, via adding logic gates in LUT section of CLB, power dissipation through controlling $V_{s s v}$ state is changed dramatically, such that when $V_{s s v}=0$, power is raised and in case of $V_{s s v}=1$, circuit goes to power gated state and power dissipation get reduced. Results also show that 
there exist linear equation among average power reduction and activity of $V_{s s v}$ in sense of time interval. In this research initially $100 \mu \mathrm{s}$ is considered as a time reference for controlling $V_{s s v}$ logic state such that, the sample circuits of CLB are tested between $10 \mu \mathrm{s}$ to $50 \mu \mathrm{s}$ out of $100 \mu \mathrm{s}$ at logic state of $V_{s s v}=1$ and remained time of $100 \mu \mathrm{s}$ at logic state of $V_{s s v}=0$. Result shows that, staying on power gated state for $10 \%$ up to $50 \%$ of total time, can reduce power dissipation, respectively $9 \%$ to $49 \%$. By presenting time controller of $V_{s s v}$ logic state, power is optimized while having a criterion of power dissipation measurement for power gated state $\left(V_{s s v}=1\right)$. In addition when $V_{s s v}=1$ and circuit is in Power gated state, logic state can be preserved and this preserving logic state, leads to reducing power dissipation.

\section{ACKNOWLEDGMENT}

The authors wish to thank Dr. Hadi Jahanirad for his technical support.

\section{REFERENCES}

[1] J. Friedrich, B. McCredie, N. James, B. Huott, B. Curran, E. Fluhr, E.Chan, G. Mittal, D. Plass, Y. Chan, S. Chu, H. Le, L. Clark, J. Ripley, S. Taylor, J. Dilullo, and M. Lanzerotti, "Design of the Power6 microprocessor,"in Proc. IEEE Int. Solid-State Circuits Conf., Feb. 2007,pp. 96-97.

[2] H. Iwai, "CMOS Technology_Year 2010 and Beyond," IEEE J. Solid-State Circuits, vol. 34, pp. 357-366, Mar. 1999.

[3] Anis, M.H.; Allam, M.W.; Elmasry, M.I., "Energyefficient noise-tolerant dynamic styles for scaled-down CMOS and MTCMOS technologies," in Very Large Scale Integration (VLSI) Systems, IEEE Transactions on , vol.10, no.2, pp.71-78, April 2002

[4] Tran, C.Q.; Kawaguchi, H.; Sakurai, T., "95\% LeakageReduced FPGA using Zigzag Power-gating, DualVTH/VDD and Micro-VDD-Hopping," in Asian SolidState Circuits Conference, 2005 , vol., no., pp.149-152, Nov. 2005

[5] L.Benini and G.D.Mecheli. "State assignment for low power dissipation" IEEE J Solid- state circuits, vol 30, no.2, pp.258-268, mar. 1995.

[6] Kursun, V.; Friedman, E.G., "Domino logic with variable threshold voltage keeper," in Very Large Scale Integration (VLSI) Systems, IEEE Transactions on , vol.11, no.6, pp.1080-1093, Dec. 2003

[7] S. Mutoh, T. Douseki, Y. Matsuya, T. Aoki, S. Shigematsu, and J. Yamada, "A 1-V power supply highspeed digital circuit technology with multithresholdvoltage CMOS," IEEE J. Solid-State Circuits, vol. 30,no. 8, pp. 847-854, Aug. 1995.

[8] S. G. Narendra and A. Chandrakasan, Eds., Leakage in Nanometer CMOS Technologies. New York: Springer, 2005.

[9] J.Semon and Y.Shin "Design and optimization of PowerGated circuits with autonomous data retention," IEEE TRANSACTION ON [VLSI]SYSTEMS ,VOL 19,no.2, pp.227-235,feb 2011

[10] H. Mair, A.Wang, G. Gammie, D. Scott, P. Royannez, S. Gururajarao, M. Chau, R. Lagerquist, L. Ho, M. Basude, N. Culp, A. Sadate, D. Wilson, F. Dahan, J. Song, B. Carlson, and U. Ko, "A 65-nm mobile multimedia applications processor with an adaptive power management scheme to compensate for variations," in Proc. Symp. VLSICircuits, Jun. 2007, pp. 224-225.

[11] Yan Lin; Fei Li; Lei He, "Routing track duplication with fine-grained power-gating for FPGA interconnect power reduction," in Design Automation Conference, 2005. Proceedings of the ASP-DAC 2005. Asia and South Pacific , vol.1, no., pp.645-650 Vol. 1, 18-21 Jan. 2005

[12] Rahman, Arifur; Das, S.; Tuan, T.; Trimberger, S., "Determination of Power Gating Granularity for FPGA Fabric," in Custom Integrated Circuits Conference, 2006. CICC '06.IEEE, vol., no., pp.9-12, 10-13 Sept. 2006

[13] Bsoul, A.A.M.; Wilton, S.J.E., "An FPGA architecture supporting dynamically controlled power gating," in Field-Programmable Technology (FPT), 2010 International Conference on, vol., no., pp.1-8, 8-10 Dec. 2010

[14] Ishihara, S.; Hariyama, M.; Kameyama, M., "A LowPower FPGA Based on Autonomous Fine-Grain Power Gating," in Very Large Scale Integration (VLSI) Systems, IEEE Transactions on, vol.19, no.8, pp.1394-1406, Aug. 2011

[15] Bsoul, A.A.M.; Wilton, S.J.E., "An FPGA with powergated switch blocks," in Field-Programmable Technology (FPT), 2012 International Conference on , vol., no., pp.8794, 10-12 Dec. 2012

[16] Goeders, J.B.; Wilton, S.J.E., "VersaPower: Power estimation for diverse FPGA architectures," in FieldProgrammable Technology (FPT), 2012 International Conference on , vol., no., pp.229-234, 10-12 Dec. 2012.

[17] Chin HauHoo; Yajun Ha; Kumar, A., "A directional coarse-grained power gated FPGA switch box and power gating aware routing algorithm," in Field Programmable Logic and Applications (FPL), 2013 23rd International Conference on , vol., no., pp.1-4, 2-4 Sept. 2013

[18] Bsoul, A.A.M.; Wilton, S.J.E.; Tsoi, K.H.; Luk, W., "An FPGA Architecture and CAD Flow Supporting Dynamically Controlled Power Gating," in Very Large Scale Integration (VLSI) Systems, IEEE Transactions on , vol.PP, no.99, pp.1-1, feb. 2015

[19] Ayorinde, Oluseyi; He Qi; Yu Huang; Calhoun, Benton H., "Using island-style bi-directional intra-CLB routing in low-power FPGAs," in Field Programmable Logic and Applications (FPL), 2015 25th International Conference on , vol., no., pp.1-7, 2-4 Sept. 2015

[20] RABAEY, J; CHANDRAKASAN, A.; NIKOLIC, B. Digital Integrated Circuits: a design perspective. 2nd Edition. Prentice Hall, 2003. ISBN: 0-13-090996-3.

[21] Bhavya Daya; Low-Power Configurable Logic Block (CLB) Design for FPGAs, http://web.mit.edu/ 2009

[22] George, V., et al., "The Design of a Low Energy FPGA," ISLPED 1999.

\section{Authors' Profiles}

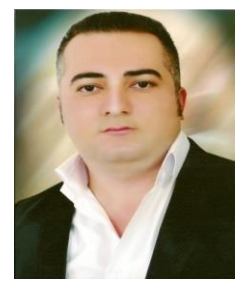

Shoaib Shokuhi was born in Sanandaj, Iran in 1986. He received his B.Sc. in electronics engineering from Mehr University, Arak, Iran in 2011 and his M.Sc. degree in electronics engineering from Islamic Azad University, Science and Research branch (Kurdistan), Tehran, Iran in 2014. His research interests include VLSI circuits Design, Embedded system ,Configurable computing and Low power Design. 


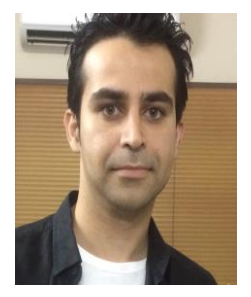

Ali Hosseini was born in Sanandaj, Iran in September 1987. He received his B.Sc. in electronics engineering from Applied Science and Technology University, Arak, Iran in 2011 and his M.Sc. degree in electronics engineering from Islamic Azad University, Science and Research branch (Kurdistan), Tehran, Iran in 2014. His research interests include VLSI circuits Design, Embedded system, Fault Tolerant computing, Configurable computing, Low Power Design, Biomedical Electronics, IOTs and sensor networks.

How to cite this paper: Shoaib Shokuhi, Ali Hosseini,"Power Optimization of Configurable Logic Block in FPGA via Controlling Logic State of Virtual Ground Voltage", International Journal of Image, Graphics and Signal Processing(IJIGSP), Vol.8, No.2, pp.45-52, 2016.DOI: 10.5815/ijigsp.2016.02.06 